\title{
A Simple Method to AMPlify Micro DISPLACEMENT
}

\author{
Yizong He and Jian-Ping Wang \\ Department of Electrical and Computer Engineering, University of Minnesota, \\ Minneapolis, MN 55455, USA
}

\begin{abstract}
A simple method to amplify the micro displacements produced by magnetostrictive effect, giant magnetostrictive, converse piezoelectric and photo strictive effect, respectively, is reported. The device consists mainly of two material rods with different coefficients of strains vs intensity of external fields, which are rigidly jointed, so that the displacements created by different rods can be added directly. In contrast with all other methods reported so far, which are all based on the principle of lever, this approach holds some unique advantages that can be applied to respond to the electromagnetic fields with high frequency because there is no friction caused by the relative motion between levers and furthermore, an ideal and smooth amplification of micro displacement can be obtained with ease in principle.
\end{abstract}

\section{KEYWORDS}

Amplification of micro displacement, Wagneto strictive effect, Giant magneto strictive effect, Converse piezoelectric effect, Photo strictive effect

\section{INTRODUCTION}

In order to gain a smooth puller for Czochralski technique of crystal growth, a novel puller was proposed in 1999, which pulling process of single crystals is not carried out by traditional gear motors, but the expansion of metal rods heated [1]. However the extremely tiny coefficient of thermal expansion of metal or alloy obliged us to look for a good approach to amplify the micro displacements, then a concise way to get big displacement was designed [2].

It is well known, that both magnetostrictive and giant magnetostrictive materials change their sizes and shapes when they are placed in a magnetic field, and such an effect is called a magnetostrctive effect. Similarly, a converse piezoelectric and electrostrictive effect occurs when some crystals are positioned in an electric field; likewise, the generation of strain induced by the irradiation of light is defined as a photostrictive effect.

All above effects produce, to a different extent, microdisplacements (expansion or contraction), which are vertical or parallel to external fields (a magnetic or electric or irradiation light field). There is a great deal of applications based on the principle that strains occur when external fields are applied on some materials. The applications relate to the aerospace or the defense industry, electronics, machinery, petroleum, textile, agricultural and other fields [3-6].

For the material of magnetostrictive effect, the magnetostrictive coefficient is essential among several parameters (magnetostrictive coefficient, mechanical energy to electrical energy conversion arte, energy density, response speed, reliability, driving mode, working frequency 
International Journal of Instrumentation and Control Systems (IJICS) Vol.6, No.2/3, July 2016

range and stability, etc.) to characterize the magnetostrictive material (such as $\mathrm{CoFe}_{2} \mathrm{O}_{4}$ ) and giant magnetostrictive material (such as $\mathrm{TbFe}_{2}, \mathrm{SmFe} 2$ (with magnetostrictive coefficient $1.753 \times 10^{-3}$,$1.560 \times 10^{-3}$,respectively). Generally speaking, the larger the magnetostrictive coefficient $d_{i j}$, the more useful the magnetostrictive material is. The relationship of strain $\varepsilon_{i}, d_{i j}$ and the external magnetic field $H_{i}$ can be depicted by the equation (1) below

$$
\left[\begin{array}{l}
\varepsilon_{1} \\
\varepsilon_{2} \\
\varepsilon_{3} \\
\varepsilon_{4} \\
\varepsilon_{5} \\
\varepsilon_{6}
\end{array}\right]=\left[\begin{array}{l}
d_{11} d_{21} d_{31} \\
d_{12} d_{22} d_{32} \\
d_{13} d_{23} d_{33} \\
d_{14} d_{24} d_{34} \\
d_{15} d_{25} d_{35} \\
d_{16} d_{26} d_{36}
\end{array}\right]\left[\begin{array}{l}
H_{1} \\
H_{2} \\
H_{3}
\end{array}\right]
$$

For the converse piezoelectrictive and electrostrictive effects can be depicted by equation (2), where $\varepsilon_{i}, d_{i j}, s_{i j}$ and $E_{j}$ presents strain, converse piezoelectristive coefficient, photostrictive coefficient and electric intensity, respectively.

$$
\left[\begin{array}{l}
\varepsilon_{1} \\
\varepsilon_{2} \\
\varepsilon_{3} \\
\varepsilon_{4} \\
\varepsilon_{5} \\
\varepsilon_{6}
\end{array}\right]=\left[\begin{array}{l}
d_{11} d_{21} d_{31} \\
d_{21} d_{22} d_{23} \\
d_{31} d_{32} d_{33} \\
d_{41} d_{42} d_{43} \\
d_{51} d_{52} d_{53} \\
d_{61} d_{62} d_{63}
\end{array}\right]\left[\begin{array}{l}
E_{1} \\
E_{2} \\
E_{3}
\end{array}\right]+\left[\begin{array}{l}
s_{11} s_{12} s_{13} \\
s_{21} s_{22} s_{23} \\
s_{31} s_{32} s_{33} \\
s_{41} s_{42} s_{43} \\
s_{51} s_{52} s_{53} \\
s_{61} s_{62} s_{63}
\end{array}\right]\left[\begin{array}{l}
E_{1}^{2} \\
E_{2}^{2} \\
E_{3}^{2}
\end{array}\right]
$$

Both the converse piezoelectric and electrostrictive materials (such as $\mathrm{BaTiO}_{3}$ and $\mathrm{Pb}\left(M g_{\frac{1}{3}} N b_{\frac{2}{3}}\right) \mathrm{O}_{3}$, respectively) can be transverse or longitudinal.

Researchers have been laboriously looking for the materials with larger magnetostrictive coefficients. The giant magnetostrictive coefficients, such as $\mathrm{TbFe}_{2}$ and $\mathrm{SmFe}_{2}$ with $1.753 \times 10^{-3}$ and $-1.560 \times 10^{-3}$ respectively, are much larger than that of conventional magnetostrictive materials. Even so, its magnitude is still not big enough, in the order of magnitude of $10^{-3}$. Therefore, the amplification of the micro-displacement is very significant for practical applications. This simple method reported here increases the displacement dozens, and even more than one hundred times, with ease. Comparing all previous ways to amplify micro displacement [7-9], although this system consists of only some mechanical rods, no relative sliding motion occurs, so that not any phase delay yields at all when the external magnetic field is alternating. Therefore, it can satisfy the amplification of displacements caused by a high frequency magnetic field. It is obvious that two displacements created by two rods of materials can be added directly. It is easy to imagine, in principle, there is no limitation to this amplification.

In order to obtain larger displacement, we generalized the basic idea proposed in the thermal expansion case. We first describehow to enlarge displacement produced by the magnetostrictive effect in detail, and then extend the design to converse piezoelectric effect, photostrictive effect cases briefly. 
International Journal of Instrumentation and Control Systems (IJICS) Vol.6, No.2/3, July 2016

\section{THE DESIGNS OF THE AMPLIFICATION}

The principle of amplification of micro displacement is shown in Fig.1. The main components are two kinds of rigid rods (1) and (2) made from magnetostrictive (or giant magnetostrictive) materials, with positive $d_{33}$ and negative $d_{33}$ (see Fig.2) connected by non-magnetostrictive rods (3) rigidly. Two magnetostrictive rods will get longer and shorter, respectively, when magnetic fields are exerted on the amplified system. The direction of the magnetic field should be decided by the properties of material rods, transverse or longitudinal magnetostriction. For the transverse magnetostrictive material, the orientation of the external field is perpendicular to the direction of the magnetic field, and for longitudinal one along the direction of magnetic field.

\section{A CONCRETE EXAMPLE}

One concrete example is given as following:TbFe2 giant magnetostrivtive alloy [10,11]with the positive magnetostrictive coefficient $+1.753 \times 10^{-3}$, was selected as the red material rods (1) (shown in Fig. 1) and $\mathrm{SmFe}_{2}$ with $-1.560 \times 10^{-3}$ the green rods (2) in the same figure [12].Let lbe red and green long rod, with $100 \mathrm{~cm}$ in length, cut both rods into 5 segments with $20 \mathrm{~cm}$ long. The net total displacements for the system, in external saturation magnetic field,can be calculated by the equation below:

$$
\begin{gathered}
\Delta l=\left[(5+1) d_{33, \mathrm{TbFe}_{2}}+5 \mid d_{33, \mathrm{SmFe}_{2} \mid}\right] l \\
=\left[6 \times 1.753 \times 10^{-3}+5\left|-1.560 \times 10^{-3}\right|\right] \times 200 \mathrm{~mm}=3.66 \mathrm{~mm}
\end{gathered}
$$

$3.66 \mathrm{~mm}$ is a macro-size that can be seen by naked eye. The size of whole device is reduced from $100 \mathrm{~cm}$ into $20 \mathrm{~cm}$, and what's more, the current to create the saturation magnetic field can be decreased considerably.

Of course, besides $\left|d_{33, S m F e_{2}}\right|$, some others $\mathrm{d}_{\mathrm{ij}}$ components also are not equal to zero. Fortunately, the rods can be placed symmetrically based on the concrete values ofd $\mathrm{i}_{\mathrm{ij}}$, so that the distortion displacements can be cancelled each other completely.

\section{Some Similar Device For Amplication}

Similarly, the red rod (1) (as shown in Fig. 3) with positive converse piezoelectric (or electrostrictive) coefficient $d_{33}$ will get longer along the vertical direction, while the green rod (2) will get shorter when the external electric field is exerted. The working principles of two electrodes (4) and (5) depend on whether the red and green rods are longitudinal or transverse. One can coordinate the relationship among positive or negative strain coefficients, longitudinal (or transverse)piezoelectric (or electrostrictive) effect, to obtain additional net displacements caused by red and green rods.

Finally, by irradiating asymmetric piezoelectric ferroelectrics (such as PLZT), with light, an electric field is caused by the photovoltaic effect. The electric field then causes a mechanical strain by the piezoelectric effect. The amplification of microdisplacement caused by the photostrictive effect is shown in Fig. 4. The red (or green) rods stand for photostrictive material with positive (or negative) transverse photostrictive coefficients $d_{33}$. Thetotal displacement equals the summation of the positive and the absolute value of the negative displacement. As the number of strain rods increase, the amount of displacements increases linearly. This effect can be applied 
International Journal of Instrumentation and Control Systems (IJICS) Vol.6, No.2/3, July 2016

to a specific bender. When the light irradiates on some photostrictive material rods which are attached to a piece of non-photostrictive plate, the bender begins to bend. When its microdisplacement is amplified by this method, the displacement amount will increase many times more than the amount by the normal way.

\section{SUMMARIES AND CONCLUSIONS}

In contrast with all other methods, up to now, to amplify microdisplacements, this simple one holds some unique advantages that can suitable to the external fields with high frequency because the joint of the rods are all rigid. Conversely, the traditional approaches, all are based on the principle of lever, consequently, the existence of friction forces between materials rods will delay the response to the external fields. It is obvious lots of times of amplification of microdisplacement can be gained with ease by assembling all kinds of material to respond to different external fields.

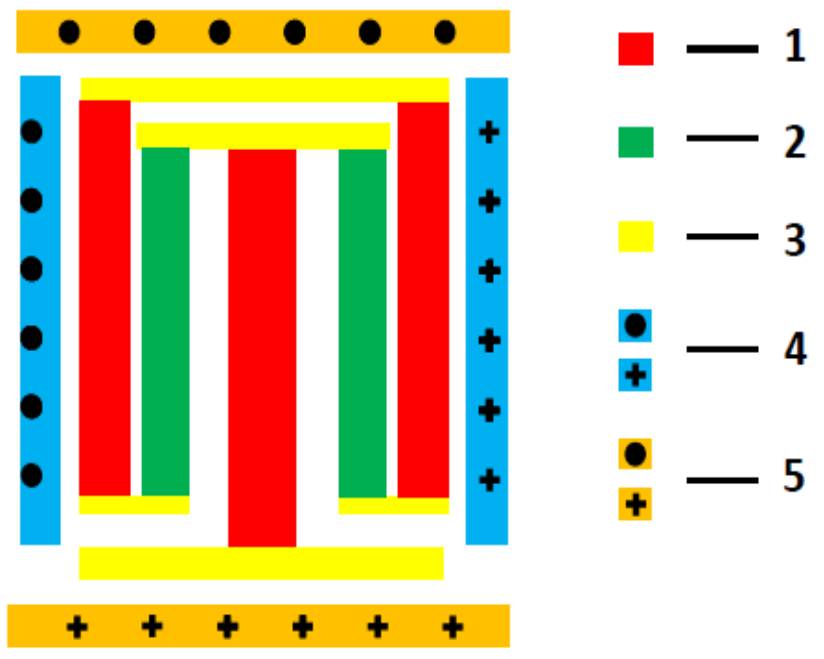

Figure. 1 The illustraction of the principle to amplify a microdisplacement caused by magnetostrictive effect

(1) Material rod with positive magnetostrictive (or giant-magnetostrictive) coefficient;

(2) material rod with negative magnetostrictive (or giant-magnetosrictive) coefficient;

(3) non-magnetostrictive material rod to connect 1 and 2;

(4) magnet to create vertical magnetic field;

(5) magnet to create horizontal magnetic field. 


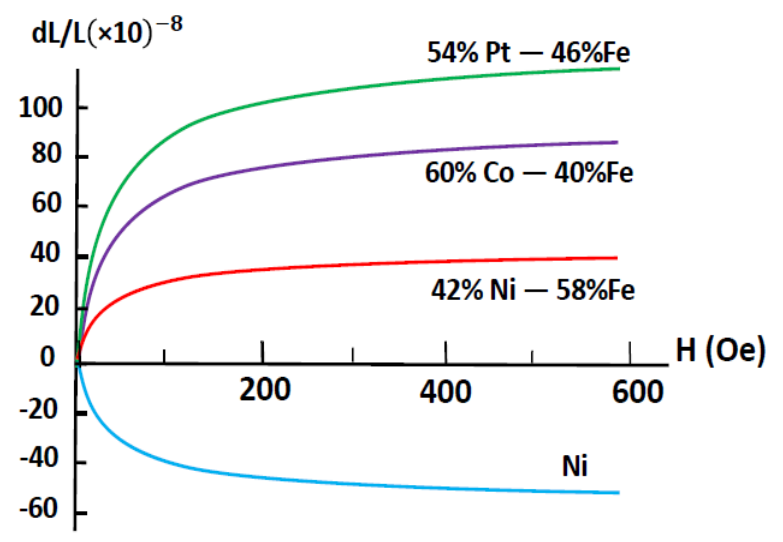

Figure.2 Dependence of the longitudinal magnetosctriction of a number of polycrystalline metals, alloys and compounds on magnetic field intensity

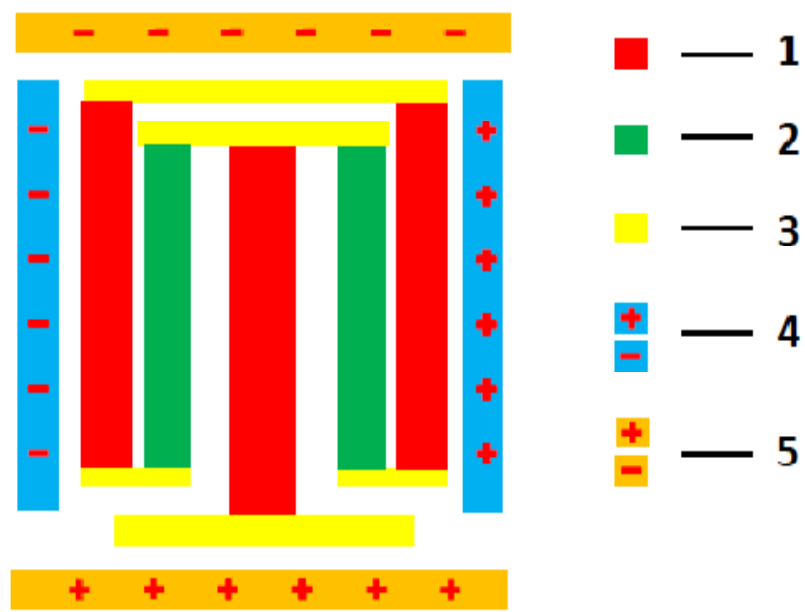

Figure. 3 The illustraction of the principle to amplify a micro-displacement caused by converse piezoelectric or electrostrictive effect

(1) material rod with a big and positive converse piezoelectric or electrostrictive coefficient;

(2) material rod with big and negative converse piezoelectric or electrostrictive coefficient;

(3) non-converse or electrostictive rod to connect (1) and (2);

(4) electrodes to create horizontal electric field;

(5) electrodes to create vertical electric field. 


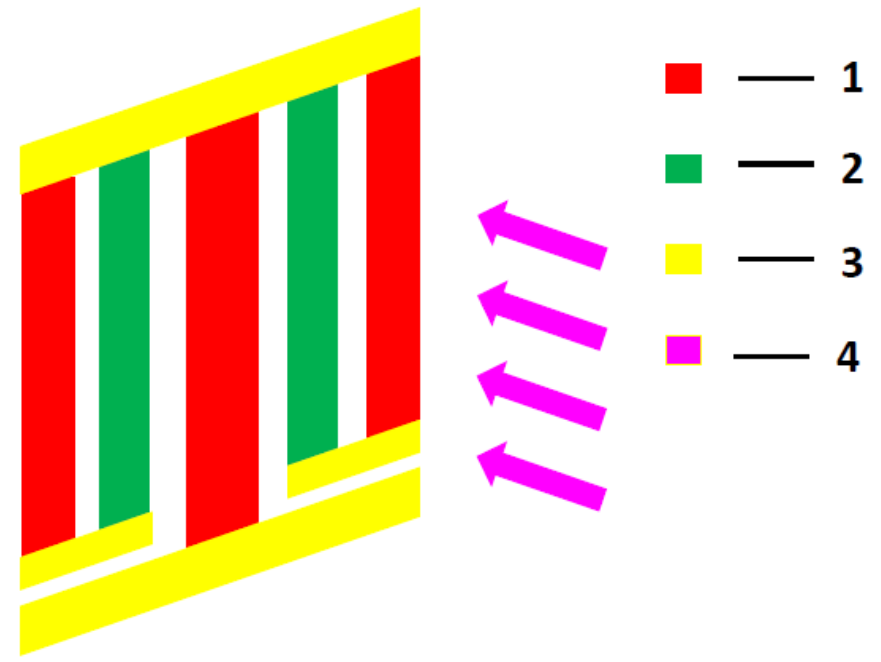

Figure. 4 The illustractin of the principle to amplify the micro-displacement caused by photosctrictive effect

(1) material rod with positive photostrictive coefficient;

(2)material rod with negative photostrictive coefficient or non-photostrictive material;

(3) non-photostrictive material to connect 1 and 2;

(4) incident light

\section{REFERENCES AND LINKS}

[1] Y. Z. He and F.Zhou(1999) Review of Scientific Instruments, 70, Iss.11, 4313

[2] P. L. Lang, Y. Z. He, F. Zhou and Z. X. Zhao(2001) Review of Scientific Instruments,72(2), 1585

[3] Zhonglei Sun, Meiying Zhao and Zidong Yin, Part Design of Giant Magnetostrictive Actutor, 2011 International Conference on Physics Science and Technology,(ICPST 2011) Physics Procedia 22,80 (2011).

[4] Yoshio Yamamoto, etc, Application of Giant Magnetostrictive Materials of Positioning Actuators, Proceedings of the 1999 IEEE/ASME, International conference on Advanced Intelligent Mechatronics, September 19-23, Altanta, USA (1999)

[5] S. Bonora, etc, Applied Physics Letters 99, 033506 (2011)

[6] Graham Flanagan, William John Metheringham, David Johnson and Brian Douglas Smith, USA invention patent authorization number: US6891286 B2 (the application number: US 10/913,582, publication date on May.10, 2005) The link to this patent is

"https://www.google.com/patents/US6891286"

[7] Matthew B. Parkinson, Proceedings of DETC'01 ASME 2001 Design Engineering Technical Conferences and Computers and information in Engineering Conferences, Pittsburgh, Pennsylvania, September 9-12, (2001) 
International Journal of Instrumentation and Control Systems (IJICS) Vol.6, No.2/3, July 2016

[8] Pengchen Xie, Weiming Yang and Yuming ding, Chinainvention patent authorization number: 101166005 B (the application day is Oct. 20, 2006, disclosed on May.19, 2010) This patent is still

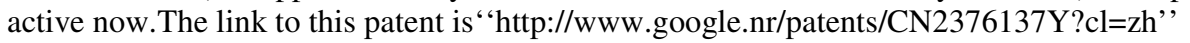

[9] Hongkang Si, Lian Ye, Jun Ye, Xiaobing Wang, Xiaolong Ye, Yue Zhou and Yiqi Jin, China invention patent, (Application number: CN 201410371832,publication date: Dec. 10 2014,publication number: CN104201932 A,publication type: application) The link to this patent is "https://www.google.com/patents/CN104201932A?cl=en\&dq=\%E7\%A3\%81\%E8\%87\%B4\%E4\%B C\%B8\%E7\%BC\%A9+\%E5\%BA\%94\%E7\%94\%A8\&ei=1KZXVbbRHcrJsAWGsIDABg”,

[10] N.C. Koon, C.M. Williams and B.N. Das(1991) Journal of magnetism and magnetic materials, vol. 100 , issues $1-3,173$

[11] Go ran Engdahl, Handbook of Giant Magnetostrictive Material, Academic Press, A Harcourt Science and Technology Company, 525 B Street, Suite 1900, San Diego, CA 92101-4495, USA, http://www.apnet.com

[12] W.J. Ren, J.L. Yang, B. Li, D. Li, X.G. Zhao and Z.D. Zhang(2009) Physica B, 404, 3410 